\title{
Research Article \\ Effects of Erroneous CSI on the Performance of Multiple-Transmit Antenna Selection
}

\author{
Mingjie Zhuang \\ College of Engineering, Huaqiao University, Quanzhou, Fujian 362021, China \\ Correspondence should be addressed to Mingjie Zhuang; mjzhuang176@163.com
}

Received 28 April 2014; Accepted 14 September 2014

Academic Editor: Thomas Kürner

Copyright (C) 2015 Mingjie Zhuang. This is an open access article distributed under the Creative Commons Attribution License, which permits unrestricted use, distribution, and reproduction in any medium, provided the original work is properly cited.

\begin{abstract}
The paper assumes that the channel state information (CSI) is available at the receiver and is known partially at the transmitter through a feedback channel. The bit error rate (BER) performance of multiple transmit antenna selection (MTAS) for the multipleinput and single-output (MISO) system with STBC (MTAS/MISO-STBC) will be investigated in detail. Meanwhile, the selection criterion that maximizes the channel Frobenius norm or minimizes the error probability of MTAS/MISO-STBC system is employed. In two cases of erroneous CSI (ECSI) and inerrable CSI (ICSI), wireless channels of the MTAS systems are modeled and their analytical expressions are derived. For the case of ICSI, the exact BER expressions of the Chernoff upper bound (CUB) for both the MISO-STBC system of full complexity and MTAS system are evaluated, respectively. Next, for the ECSI case, a comprehensive analytical expression of BER CUB for the same system is derived in detail. Finally, extensive Monte-Carlo simulations are presented to support and validate our numerical analysis proposed in this paper. Both the simulating results and the numerical results show that MTAS can reduce effectively the effect of the erroneous CSI.
\end{abstract}

\section{Introduction}

Providing reliable wireless communication has obtained the great attention of communication community in recent years. This stems from the fact that in a wireless environment, unlike other applications, achieving reliable communication is much more challenging due to the possibility that received signals from multi-path may add destructively, which, consequently, results in a serious performance degradation. Multiple-transmit multiple-receive antenna (also referred to as multiple-input multiple-output, MIMO) systems are increasingly important because the multiple antenna technology can significantly improve wireless link performance. Degrees of freedom afforded by the multiple antennas may be used to increase reliability through space-time diversity techniques [1-3] and/or to increase data rate through spatial multiplexing techniques [4-9]. However, among the implications of employing multiple antennas are the considerable increase in the cost of the additional hardware required in the form of RF chains, as well as the physical limitation of wireless devices. In an effort to overcome these problems, while utilizing the advantages of using multiple antennas, several papers [10-22] have appeared in the literature in which the notion of antenna selection was introduced for space-time coding (STC). Antenna subset selection, where transmission and/or reception is performed through a selection of the total available antennas is a powerful solution that reduces the need for multiple analog chains yet retains many diversity benefits. The main idea of multiple antenna selection (MAS) is to employ a limited number of analog chains that are adaptively switched to a subset of the available antennas.

Early works in MAS have focused on the multipleinput single-output (MISO) channel or the single-input multiple-output (SIMO) channel. They mainly investigated MAS from the point of view of the diversity and then chose the best limited number of available antennas at the receiver and/or transmitter. The receiver and/or transmitter performs maximal ratio combining (MRC) and/or the hybrid selection and MRC approach [23]. Since [10, 11] introduced the notion of optimal antenna subset selection, recently, there has been an explosion of interests in the application of antenna subset selection techniques to MIMO systems [1221]. In [10], it has shown that antenna selection techniques applied to low-rank channels could increase capacity. In [11], 
a criterion for selecting antenna subsets to maximize the channel capacity was presented. A fast selection algorithm based on "water-pouring" type idea was presented in [11]. In [12], an upper bound on the capacity of a system with the antenna subset selection was derived. In [13], an antenna selection algorithm for minimizing error rate (ER) in spatial multiplexing systems with linear receivers was presented. In [14], exact expressions for the average signal-to-noise ratio (SNR) increase through antenna selection with Alamouti code transmission were presented. Maximization of the capacity requires first the computation of the capacity value of any possible combination of $L$ processed data streams out of the possible $N$ received signals. This requires the evaluation of $C_{N}^{L}$ determinants, which is computationally prohibitive, especially if the channel changes rapidly and the antenna selection has to be reevaluated frequently. A suboptimal algorithm that does not need to perform an exhaustive search over all possible subsets was proposed in [15]. In [16], a fast antenna subset selection in MIMO system was introduced. Other works on MIMO antenna selection were also reported in [17-21] gave an overview.

In recent years, there are a few papers aiming at the MAS systems with space-time coding [22, 24-27]. In [22], the authors considered antenna selection at the transmitter (with full knowledge of the channel statistics) or at the receiver for STBC with particular emphasis on the Alamouti scheme. In [24], a study of the impact of antenna selection on the pairwise error probability was conducted. This work shows that, for full-rank space-time trellis coding (STTC) over quasi-static fading channels, the diversity order is maintained as that of the full-complexity system. Otherwise, the diversity order reduces and becomes dependent upon the number of selected antennas. In [25], the authors designed spacetime codes for two transmit antennas based on the pairwise error probability that performs better when antenna selection at the receiver is used. Antenna selection algorithms at the transmitter were studied in [26] when the Alamouti code and power allocation are implemented using the feedback CSI and considering errors in the information feedback to the transmitter. Other works relate to antenna selection for STC also can be found in [27-29].

Quasi-orthogonal space-time block codes (QSTBC) [30, 31] get code rate equal one at the expense of the loss of orthogonality, so its diversity gain has slighter losses than STBC's, due to the received signals being pairwise orthogonal, rather than completely orthogonal. Therefore the decoding complexity of QSTBC systems at the receiver is higher than STBC, and the error rate performance is also declined. Although references [32-34] introduced the constellation rotation technology which can achieve the same diversity gain with STBC, its decoding is still complicated at the receiver, and the transmitter is allowed to have partial knowledge of the channel. Although BLAST systems own the high transmission rate, its anti-interference ability is poor; it also requires that the receiver antenna number is greater than the transmitter. In addition, linear dispersion code (LDC) $[35,36]$ can indeed handle any configuration of transmit and receive antennas and that subsumes both V-BLAST and many proposed space-time block codes, for example, STBC, QSTBC, and so forth, as special cases. Up to now, STBC, QSTBC, and BLAST are still a kind of attractive linear space-time block codes. Comprehensive consideration of various factors, although we cannot construct an STBC with transmission rate equal to one for more than two transmit antennas, we believe that STBC is a promising technology and will be widely used at a period of the future. Therefore, this paper focuses on researching STBC systems.

In terms of BER performance for MTAS/MISO-STBC systems, difficulties of the theoretical investigation for the MAS systems are to model order statistics characteristic of selected wireless channels. The same as [22], both [27, 28] investigated directly order statistics characteristic of magnitude of squared channel fading coefficients utilizing order statistics in [37], and their marginal probability density functions (pdf) were derived. However, the exact closed-form expression of system bit-error rate (BER) performance using the marginal pdf cannot be derived, for example, the average output SNR of system only given by [22]. Similar to [25], in [27], the marginal pdf was upper-bounded. Comparing with the true BERs, this bound is quite loose in all the range of SNR. Although the authors have considered the power allocation and CSI feedback error in [26], the shortcoming is the specific paradigm of 2 out of 3 at the transmitter and one receive antenna only discussed. An exact BER expression was presented in [28] based on Alamouti scheme for 2 out of $N$ at the transmitter and $M$ receive antenna. However, the coefficients of the polynomial expansion cannot be presented explicitly, and the transmitter requires all knowledge of CSI.

This paper concentrates on antenna subset selection at the transmitter for independent and identically distributed (i.i.d.) quasi-static flat Rayleigh fading channels. For a MISO system equipped with $N$ transmit antennas and single receive antenna (without loss of generality) with encoding STBC, the transmitter chooses arbitrary $n$ out of $N$ available transmit antennas, denoted by $(N, n)$, typically $n \leq N$. The selected antennas are those with the largest instantaneous SNR. This is achieved by comparing the magnitude square of the fading coefficient at each transmit antenna and selecting the best $n$ out of them. The adopted selection criterion is clearly optimal in the sense that it maximizes the output SNRs at the receiver. In practical scenarios, it is not feasible to assume that the best antenna subset can always be selected owing to channel estimation errors, feedback delay, and feedback errors. Therefore, the impact of imperfect antenna selection subsets on system performance should be investigated. The paper assumes that the CSI is perfect at the receiver and is imperfect at the transmitter through a feedback channel. For the case of the ECSI, once CSI feedback error exists, we assume that imperfect CSI only leads to exchange of arbitrary two ordinal numbers $i$ and $j, 1 \leq i \neq j \leq$ $N$, in the squared channel fading coefficient (SFC) vector $\mathbf{X}_{N}=\left\{x_{1}, x_{2}, \ldots, x_{N}\right\}$. A model is developed by the order statistics channel for MTAS/MISO-STBC system and derive its order statistics analytical expression, namely, the marginal probability density function ( $p d f$ ) of squared magnitudes of the fading coefficients corresponding antenna selection subset. This paper focuses on the BER performance Chernoff 


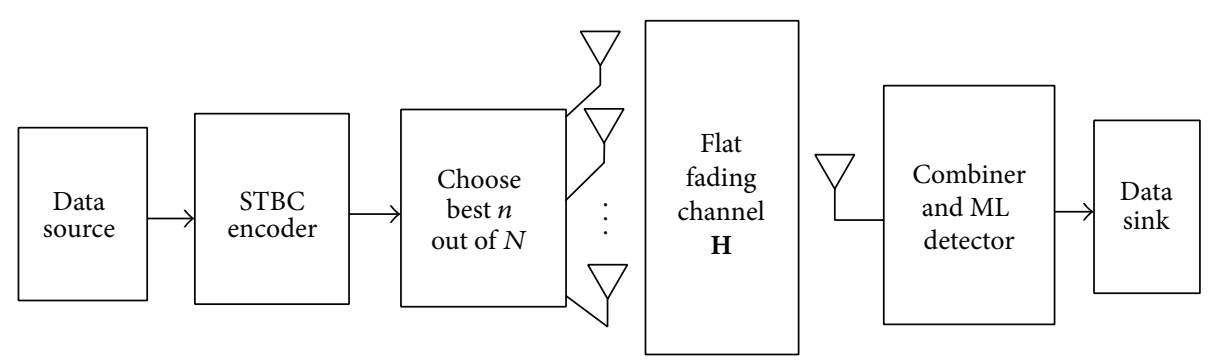

Figure 1: A block diagram of the MTAS/MISO-STBC system.

upper bound (CUB) analysis of STBC with imperfect MTAS. Considering the three cases of STBC with code rate $R_{c}=$ $1,1 / 2$ and $3 / 4$, the paper derives a more accurate analytical expression of BER CUB for MTAS/MISO-STBC system. Additionally, we also calculate the exact expression of BER CUB for the ICSI case over the MTAS/MISO-STBC system and the full complexity (no antenna selection) MISO system, respectively. Finally, we present that the extensive MonteCarlo simulations validate our theoretical analysis and verify our numerical analyses proposed in this paper. The results show that employing MAS technique can mitigate effectively the effects of the ECSI.

The paper is organized as follows. In Section 2, MISO system models for the cases of ECSI and ICSI are described and order statistical characteristics of the selection channel are derived. In Section 3, we summarize the BER CUB of full complexity MISO-STBC system. Then the BER CUB expressions of both the ICSI and the ECSI are derived, respectively. Several numerical results and simulation results that support our analyses are presented in Section 4. We make the conclusions in Section 5.

\section{System Model and Channel Characteristic of MTAs}

2.1. System Model. A block diagram of system under consideration is shown in Figure 1, which models a wireless communications system that employs $N$ antennas at the transmitter side and one antenna at the receiver side. If the incoming data have been encoded with a STBC encoder, the output of the encoder is then fed into a serial-toparallel converter that converts the input streams into $N$ parallel streams. The resulting $N$ streams are transmitted from the $N$ transmit antennas simultaneously. Blocks that involve modulation, demodulation, and so forth have been suppressed from the figure due to their irrelevancy in the analysis.

At the receiver, after demodulation, matched filtering, and sampling, the signal $r(l)$ received by antenna at time $l T$ ( $T$ denotes a symbol period) is given by

$$
r(l)=\sum_{i=1}^{N} h_{i}(l) c_{i}(l)+\eta(l),
$$

where $c_{i}(l)$ is the transmitted symbol from the $i$ th antenna at time $l T$; the noise $\eta(l)$ at time $l T$ is modeled as independent samples of a zero-mean complex Gaussian random variable with variance $N_{0} / 2$, and $N_{0}$ is single side Gaussian power spectral density. Coefficients $h_{i}(l)$ for $i=1,2, \ldots, N$ model fading between the $n$th transmit antenna and the receive antenna are assumed to be complex Gaussian random variables with variance $\sigma^{2}=0.5$ per dimension. We assume a quasi-static flat fading channel, that is, fading coefficients $h_{i}(l)$ are constant during a frame and vary from one frame to another and the fading subchannels are independent.

\subsection{Selective Channel Characteristic}

2.2.1. Inerrable CSI (ICSI). In this case, the channel state information (CSI) is perfectly known at both the receiver and the transmitter. We will examine the arbitrary number $n$ out of available transmit number $N$, namely, $(N, n)$, where $n \leq N$, for a MTAS/MISO-STBC system. Let $x_{i}=\alpha_{i}^{2}$, where $\alpha_{i}$ denotes the magnitude of channel fading coefficients $h_{i}$, and $x_{i}$ is defined as squared channel fading coefficient (SFC). The SFCs between $N$ transmit antennas and the receive antenna are expressed by vector $\mathbf{X}_{N}=\left\{x_{1}, x_{2}, \ldots, x_{N}\right\}$. Without a loss of generality, we have arranged the vector $\mathbf{X}_{N}$ in descending order, namely, $\infty \geq x_{1} \geq x_{2}, \ldots, \geq x_{N} \geq 0$. Therefore, for the $(n, N)$ system, we will choose the best $n$ SFCs and abandon the worst $(N-n)$ SFCs. Similarly, let the $n$ SFCs be denoted by vector $\mathbf{X}_{n}=\left\{x_{1}, \ldots, x_{n}\right\}$. In the following section, we will derive the marginal probability density function (pdf) of the best $n$ SFCs $\mathbf{X}_{n}$.

Applying order statistics [37], the joint pdf of $\mathbf{X}_{N}$ then is given by

$$
g\left(\mathbf{X}_{N}\right)=N ! \rho^{-2 N} e^{-\sum_{i=1}^{N} x_{i} / \rho^{2}}
$$

For a $(N, n)$ MTAS/MISO-STBC system, the marginal pdf of $\mathbf{X}_{n}$ is as follows:

$$
\begin{aligned}
g\left(\mathbf{X}_{n}\right)= & \frac{N !}{\rho^{2 N}} \int_{0}^{x_{n}} \int_{0}^{x_{n+1}} \cdots \int_{0}^{x_{N-1}} e^{-\sum_{i=1}^{N} x_{i} / \rho^{2}} d x_{n+1} \cdots d x_{N} \\
= & \frac{N !}{\rho^{2 N}} e^{-\sum_{i=1}^{n} x_{i} / \rho^{2}} \\
& \times \int_{0}^{x_{n}} \int_{0}^{x_{n+1}} \cdots \int_{0}^{x_{N-1}} e^{-\sum_{i=n+1}^{N} x_{i} / \rho^{2}} d x_{n+1} \cdots d x_{N} .
\end{aligned}
$$


Performing $(N-n)$ times integral in (3), we thus obtain the general expression of the marginal pdf of $\mathbf{X}_{n}$ for the $(N, n)$ system as

$$
g\left(\mathbf{X}_{n}\right)=\frac{N ! \rho^{-2 n}}{(N-n) !} e^{-\sum_{i=1}^{n} x_{i} / \rho^{2}}\left(1-e^{-x_{n} / \rho^{2}}\right)^{N-n}
$$

2.2.2. Erroneous CSI (ECSI). Since existing CSI feedback error, the transmitter selects possibly arbitrary $n$ out of $N$ transmit antennas to send the information. Here we assume that imperfect CSI only leads to exchange of arbitrary two ordinal numbers $i$ and $j, 1 \leq i \neq j \leq N$, in the vector $\mathbf{X}_{N}$. A total of $N(N-1) / 2$ possible selection subsets of transmit antenna can be used for transmission. For a $(N, n)$ MTAS/MISO-STBC system, if exchange between ordinal numbers $i$ and $j$ takes place both among the best $n$ transmit antennas $\mathbf{X}_{n}$ and among the worst $(N-n)$ transmit antennas $\left\{x_{n+1}, \ldots, x_{N}\right\}$, the resulting transmit antenna subset $\mathbf{X}_{n}^{\prime}$ does not affect the performance of $(N, n)$ system and the order statistics characteristics of the selective channel. They have $n(n-1) / 2$ and $(N-n)(N-n-1) / 2$ possible subsets of transmit antenna selection, respectively. However, if the exchange takes place between ordinal number $i$ of the best $n$ transmit antennas $\mathbf{X}_{n}(1 \leq i \leq n)$ and the ordinal number $j$ of the worst $(N-n)$ transmit antennas $\left\{x_{n+1}, \ldots, x_{N}\right\}(n+1 \leq j \leq N)$, then the resulting transmit antenna subset $\mathbf{X}_{n}^{\prime}$ is to affect the performance of $(N, n)$ system and order statistics characteristics of the selective channel. There are $n(N-n)$ possible subsets of transmit antenna selection. According to order statistics theory [37], following that we can derive the marginal pdf of the resulting $\mathbf{X}_{n}^{\prime}\left\{x_{1}, x_{2}, \ldots, x_{i-1}, x_{i+1}, \ldots, x_{n-1}, x_{n}, x_{j}\right\}$, in which exchange between ordinal number $i \in[1, n]$ and ordinal number $j \in[n+1, N]$ will affect order statistics characteristics of the selected channel. According to the range of $i$ values, in the following section, we will present the marginal pdf of the resulting selection subset $\mathbf{X}_{n}^{\prime}$ in three cases.

In the process period of a frame, we assume that the correct descending order of the $\mathbf{X}_{N}$ is given by

$$
\begin{aligned}
x_{1} & \geqslant x_{2} \geqslant \cdots \geqslant x_{n-2} \geqslant x_{n-1} \geqslant x_{n} \\
& \geqslant x_{n+1} \geqslant x_{n+2} \geqslant \cdots x_{j} \geqslant \cdots \geqslant x_{N} .
\end{aligned}
$$

Let $j \in[n+1, N]$. Applying (4), we can obtain the marginal pdf of random variables (RVs) $\left\{x_{1}, x_{2}, \ldots, x_{n-1}, x_{n}, \ldots, x_{j}\right\}$ as follows:

$$
f\left(x_{1}, x_{2}, \ldots, x_{j}\right)=\frac{N ! \rho^{-2 j}}{(N-j) !} e^{-\sum_{k=1}^{j} x_{k} / \rho^{2}}\left(1-e^{-x_{j} / \rho^{2}}\right)^{N-j} .
$$

Let $J=(j-1)-n$, and $y_{r}=x_{n+r}$, where the subscripts $r=$ $1,2, \ldots, J$ are the RVs vector $\left\{x_{n+1}, x_{n+2}, \ldots, x_{n+r}, \ldots, x_{j-1}\right\}$. For integral simplicity, they can be denoted by RVs vector
$\mathbf{Y}_{J}=\left\{y_{1}, y_{2}, \ldots, y_{r}, \ldots, y_{J}\right\}$. Thus the analytical expression of marginal pdf of the RVs vector $\left\{\mathbf{X}_{n}, x_{j}\right\}$ is calculated by

$$
\begin{aligned}
f\left(\mathbf{X}_{n}, x_{j}\right) \\
=\frac{N ! \rho^{-2(n+1)}}{(N-j) !} e^{-\left(x_{j}+\sum_{k=1}^{n} x_{k}\right) / \rho^{2}}\left(1-e^{-x_{j} / \rho^{2}}\right)^{N-j} \\
\quad \times \int_{x_{j}}^{x_{n}} \int_{x_{j}}^{y_{1}} \cdots \int_{x_{j}}^{y_{J-1}} e^{-\sum_{r=1}^{J} y_{r} / \rho^{2}} \times \rho^{-2 J} d y_{1} d y_{2} \cdots d y_{J-1} \\
=\frac{N ! \rho^{-2(n+1)}}{(N-j) ! J !} e^{-\left(x_{j}+\sum_{k=1}^{n} x_{k}\right) / \rho^{2}}\left(1-e^{-x_{j} / \rho^{2}}\right)^{N-j} \\
\quad \times\left(e^{-x_{j} / \rho^{2}}-e^{-x_{n} / \rho^{2}}\right)^{J} .
\end{aligned}
$$

Substituting $J=j-n-1$ into the above equation, the marginal pdf of vector $\left\{\mathbf{X}_{n}, x_{j}\right\}$ becomes

$$
\begin{aligned}
f\left(\mathbf{X}_{n}, x_{j}\right)= & \frac{N ! \rho^{-2(n+1)}}{(N-j) !(j-n-1) !} \\
& \times e^{-\left(x_{j}+\sum_{k=1}^{n} x_{k}\right) / \rho^{2}}\left(1-e^{-x_{j} / \rho^{2}}\right)^{N-j} \\
& \times\left(e^{-x_{j} / \rho^{2}}-e^{-x_{n} / \rho^{2}}\right)^{j-n-1} .
\end{aligned}
$$

Since the exchange between ordinal number $i \in[1, n]$ and ordinal number $j \in[n+1, N]$ will affect order statistics characteristics of the selected channel, therefore, in the following section, we will discuss, respectively, the marginal pdf of the RVs $\left\{x_{1}, x_{2}, \ldots, x_{i-1}, x_{i+1}, \ldots, x_{n-1}, x_{n}, x_{j}\right\}$ in three kinds of cases.

(I) $1<i<n$. Employing (8), the marginal pdf of $\left\{x_{1}, x_{2}, \ldots\right.$, $\left.x_{i-1}, x_{i+1}, \ldots, x_{n-1}, x_{n}, x_{j}\right\}$ is attained by

$$
\begin{aligned}
f( & \left.x_{1}, x_{2}, \ldots, x_{i-1}, x_{i+1}, \ldots x_{n}, x_{j}\right) \\
= & \int_{x_{i-1}}^{x_{i+1}} f\left(x_{1}, x_{2}, \ldots, x_{i}, \ldots x_{n}, x_{j}\right) d x_{i} \\
= & \frac{N ! \rho^{-2 n}}{(N-j) !(j-n-1) !} \times e^{-\left(x_{j}+\sum_{k=1, k \neq i}^{n} x_{k}\right) / \rho^{2}}\left(1-e^{-x_{j} / \rho^{2}}\right)^{N-j} \\
& \times\left(e^{-x_{j} / \rho^{2}}-e^{-x_{n} / \rho^{2}}\right)^{j-n-1} \times\left(e^{-x_{i+1} / \rho^{2}}-e^{-x_{i-1} / \rho^{2}}\right) .
\end{aligned}
$$


(II) $i=1$. When exchange between $x_{1}$ and $x_{j}$ happens, thus the marginal pdf of $\left\{x_{2}, \ldots, x_{n}, x_{j}\right\}$ is given by

$$
\begin{aligned}
f( & \left.x_{2}, \ldots, x_{n}, x_{j}\right) \\
= & \int_{x_{2}}^{\infty} f\left(x_{1}, x_{2}, \ldots, x_{n}, x_{j}\right) d x_{1} \\
= & \frac{N ! \rho^{-2 n}}{(N-j) !(j-n-1) !} \times e^{-\left(x_{j}+\sum_{k=2}^{n} x_{k}\right) / \rho^{2}}\left(1-e^{-x_{j} / \rho^{2}}\right)^{N-j} \\
& \times\left(e^{-x_{j} / \rho^{2}}-e^{-x_{n} / \rho^{2}}\right)^{j-n-1} \times e^{-x_{2} / \rho^{2}} .
\end{aligned}
$$

(III) $i=n$. When exchange between $x_{n}$ and $x_{j}$ happens, we can obtain the marginal pdf of $\mathbf{X}_{n-1}$ and $x_{j}$ as

$$
\begin{aligned}
f( & \left.x_{1}, x_{2}, \ldots, x_{i}, \ldots x_{n-1}, x_{j}\right) \\
= & \int_{x_{j}}^{x_{n-1}} f\left(x_{1}, x_{2}, \ldots, x_{i}, \ldots, x_{n-1}, x_{n}, x_{j}\right) d x_{n} \\
= & \frac{N ! \rho^{-2 n}}{(N-j) !(j-n) !} \times e^{-\left(x_{j}+\sum_{k=1}^{n-1} x_{k}\right) / \rho^{2}}\left(1-e^{-x_{j} / \rho^{2}}\right)^{N-j} \\
& \times\left(e^{-x_{j} / \rho^{2}}-e^{-x_{n-1} / \rho^{2}}\right)^{j-n} .
\end{aligned}
$$

\section{Performance Bound Analysis of ECSI for MTAs}

3.1. BER Performance Bound of Full Complexity MISO-STBC System. Assume binary phase-shift keying (BPSK) modulation, maximum-likelihood (ML) decoder, and the channel state information (CSI) are available at the receive side. For $N$ transmit antennas and signal receive antenna system employing orthogonal space-time block coding, we assume that all $N$ transmit antennas transmit equal power. Thus the system conditional BER conditioned on $\mathbf{X}_{N}$ is then expressed as [38]

$$
P_{e}\left(\gamma_{N, R_{c}}^{\mathrm{STBC}} \mid \mathbf{X}_{N}\right)=Q\left(\sqrt{2 C_{N, R_{c}} \gamma_{b} \sum_{i=1}^{N} x_{i}}\right),
$$

where $\gamma_{b}=E_{b} / N_{0}, E_{b}$ is energy per bit, and the term is as follows:

$$
C_{N, R_{c}}= \begin{cases}1 & N=2, R_{c}=1 \\ 2 & N>2, R_{c}=\frac{1}{2} \\ 1 & N=3,4, R_{c}=\frac{3}{4},\end{cases}
$$

where $R_{c}$ denotes code rate of STBC. In a Rayleigh fading channel, the pdf of $x_{i}$ is given by [38]

$$
f\left(x_{i}\right)=\frac{1}{\rho^{2}} e^{x_{i} / \rho^{2}}, \quad x_{i} \geq 0,
$$

where $\rho^{2}=2 \sigma^{2}$. Due to $\mathbf{X}_{N}$ being independent and identically distributed (i.i.d), their joint pdf can be shown to be

$$
f\left(\mathbf{X}_{N}\right)=\prod_{i=1}^{N} f\left(x_{i}\right)=\frac{\exp \left(\sum_{i=1}^{N} x_{i} / \rho^{2}\right)}{\rho^{2 N}} .
$$

To compute the average in (12), we need to average the expression in (12) with respect to $\mathbf{X}_{N}$. Combining (12) and (15), the BER calculating formula can be shown to be

$$
\begin{aligned}
& \bar{P}_{e, \text { MPSK }}^{\text {STBC-MISO }}\left(\gamma_{b}\right) \\
& =\underbrace{\int_{0}^{\infty} \cdots \int_{0}^{\infty} Q\left(\sqrt{2 C_{N, R_{c}} \gamma_{b} \sum_{i=1}^{N} x_{i}}\right) \times \frac{e^{-\sum_{i=1}^{N} x_{i} / \rho^{2}}}{\rho^{2 N} d x_{1} \cdots d x_{N}} .}_{N} .
\end{aligned}
$$

Obviously, it is quite difficult to integrate Equation (16). We utilize the Chernoff upper bound of Gaussian $Q$ function, $Q(x) \leq(1 / 2) e^{-x^{2} / 2}[38]$. Consequently, the expression in (16) can be upper-bounded as

$$
\begin{gathered}
\bar{P}_{e, \text { MPSK }}^{\text {STBC-MISO }}\left(\gamma_{b}\right) \\
\leq \frac{1}{2 \rho^{2 N}} \underbrace{\int_{0}^{\infty} \cdots \int_{0}^{\infty} \exp }_{N}\left(-C_{n, R_{c}} \gamma_{b} \sum_{i=1}^{N} x_{i}\right) \\
\quad \times e^{-\sum_{i=1}^{N} x_{i} / \rho^{2}} d x_{1} \cdots d x_{N} .
\end{gathered}
$$

By integrating the right side of the above inequality with respect to $N$ RVs in the vector $\mathbf{X}_{N}$, we can get the theoretical expression of bit error rate (BER) Chernoff upper bound (CUB) as follows:

$$
\bar{P}_{e, \text { MPSK }}^{\text {STBC-MISO }}\left(\gamma_{b}\right) \leq \frac{1}{2\left(C_{n, R_{c}} \rho^{2} \gamma_{b}+1\right)^{N}} .
$$

3.2. ICSI BER Performance for $(N, n)$ MTAS. In the case of ICSI, assuming that system all antennas transmit equal power, we will analyze the BER CUB for the $(N, n)$ MTAS/MISOSTBC system. Applying (4) the expression of the marginal pdf of $\mathbf{X}_{n}$, the calculate formula of BER is given by

$$
\begin{aligned}
\bar{P}_{e b, \text { ICSI }}^{(N, n)}\left(\gamma_{b}\right) & \int_{0}^{\infty} \int_{x_{n}}^{\infty} \cdots \int_{x_{3}}^{\infty} \int_{x_{2}}^{\infty} Q\left(\sqrt{2 C_{n, R_{c}} \gamma_{b} \sum_{i=1}^{n} x_{i}}\right) \\
& \times g\left(x_{1}, x_{2}, \ldots, x_{n}\right) d x_{1} d x_{2} \cdots d x_{n} \\
= & \int_{0}^{\infty} \int_{x_{n}}^{\infty} \cdots \int_{x_{3}}^{\infty} \int_{x_{2}}^{\infty} Q\left(\sqrt{2 C_{n, R_{c}} \gamma_{b} \sum_{i=1}^{n} x_{i}}\right) \\
& \times \frac{N ! \rho^{-2 n}}{(N-n) !} e^{-\sum_{i=1}^{n} x_{i} / \rho^{2}}\left(1-e^{-x_{n} / \rho^{2}}\right)^{N-n} d x_{1} d x_{2} \cdots d x_{n} .
\end{aligned}
$$


Let $A_{b}=C_{n, R_{c}} \rho^{2} \gamma_{b}+1$. Using CUB, the above equation can be rewritten as:

$$
\begin{aligned}
\bar{P}_{e b, \text { ICSI }}^{(N, n)}\left(\gamma_{b}\right) & \\
\leq \frac{N !}{2(N-n) !} \int_{0}^{\infty} \int_{x_{n}}^{\infty} \cdots \int_{x_{3}}^{\infty} \int_{x_{2}}^{\infty} & {\left[\rho^{-2 n} \times e^{-A_{b} \sum_{i=1}^{n} x_{i} / \rho^{2}}\right.} \\
& \left.\times\left(1-e^{-x_{n} / \rho^{2}}\right)^{N-n}\right] \\
& \times d x_{1} d x_{2} \cdots d x_{n} .
\end{aligned}
$$

To integrate the right side of inequality with respect to $x_{1}$, we can obtain

$$
\begin{aligned}
\frac{N !}{2(N-n) !} \int_{0}^{\infty} \cdots & \int_{x_{4}}^{\infty} f_{1}\left(x_{n}\right) \times \rho^{-2(n-2)} \times e^{-\left(A_{b} \sum_{i=3}^{n} x_{i}\right) / \rho^{2}} \\
\times & \left\{\int_{x_{3}}^{\infty} \frac{e^{-A_{b} x_{2} / \rho^{2}}}{\rho^{2}} \times \frac{1}{A_{b}} \times e^{-A_{b} x_{2} / \rho^{2}} d x_{2}\right\} \\
\times & d x_{3} \cdots d x_{n} .
\end{aligned}
$$

Similarly, we integrate continuously $(n-2)$ times with respect to $x_{2}, x_{3}, \ldots, x_{n-1}$ in the above equation, and we have

$$
\begin{aligned}
\frac{N !}{2(N-n) !} \int_{0}^{\infty} & \left(1-e^{-x_{n} / \rho^{2}}\right)^{N-n} \times \frac{e^{-A_{b} x_{n} / \rho^{2}}}{\rho^{2}} \times \frac{1}{(n-1) ! A_{b}^{n-1}} \\
& \times e^{-(n-1) A_{b} x_{n} / \rho^{2}} d x_{n} .
\end{aligned}
$$

Applying binomial expansion, we get

$$
\left(1-e^{-x_{n} / \rho^{2}}\right)^{N-n}=\sum_{k=0}^{N-n}\left\{C_{N-n}^{k} \times(-1)^{k} \times e^{-k x_{n} / \rho^{2}}\right\},
$$

where the term $C_{m}^{n}=(m ! /(n !(m-n) !))$ denotes combination. Substituting the binomial expansion into (22), we arrive at

$$
\begin{aligned}
& \frac{N !}{2(N-n) !(n-1) ! A_{b}^{n-1}} \\
& \quad \times \sum_{k=0}^{N-n} \int_{0}^{\infty}(-1)^{k} C_{N-n}^{k} \times \frac{1}{\rho^{2}} \times e^{-\left(n A_{b}+k\right) x_{n} / \rho^{2}} d x_{n} .
\end{aligned}
$$

Integrating with respect to $x_{n}$, finally, we obtain the theoretical expression of the BER CUB as follows:

$$
\begin{aligned}
& \bar{P}_{e b, \text { ICSI }}^{(N, n)}\left(\gamma_{b}\right) \\
& \quad \leq \frac{N !}{2(n-1) ! A_{b}^{n-1}} \sum_{k=0}^{N-n} \frac{(-1)^{k}}{\left(n A_{b}+k\right) \times k ! \times(N-n-k) !} .
\end{aligned}
$$

Substituting $A_{b}$ into (25), we have

$$
\begin{aligned}
\bar{P}_{e b, \text { ICSI }}^{(N, n)}\left(\gamma_{b}\right) & \frac{N !}{2(n-1) !\left(C_{n, R_{c}} \rho^{2} \gamma_{b}+1\right)^{n-1}} \\
\leq & \times \sum_{k=0}^{N-n} \frac{(-1)^{k}}{\left[n\left(C_{n, R_{c}} \rho^{2} \gamma_{b}+1\right)+k\right] \times k ! \times(N-n-k) !} .
\end{aligned}
$$

For the specific case of $N=n$, namely, no antenna selection, (26) will degrade to (18).

3.3. ECSI BER Performance Bound of MTAs. According to the discussion above in Section II.B, if the CSI at transmitter side has imperfect knowledge, we will combine the three marginal pdfs of (9), (10), and (11) with (12) and derive the BER performance bound of the $(N, n)$ MTAS/MISO-STBC system.

(I) Exchange between $x_{i}(1<i<n)$ and $x_{j}(j \geqslant n+1)$. In this case, applying (9), thus the integral formula of BER for the $(N, n)$ MTAS/MISO-STBC system is clearly given by

$$
\begin{aligned}
\bar{P}_{e b, \mathrm{ECSI}, i j}^{(N, n)}\left(\gamma_{b}\right) & \\
=\int_{0}^{\infty} \int_{x_{j}}^{\infty} \int_{x_{n}}^{\infty} \cdots \int_{x_{i+1}}^{\infty} \int_{x_{i-1}}^{\infty} \cdots \int_{x_{2}}^{\infty} Q & \left(\sqrt{2 C_{n, R_{c}} \gamma_{b}\left(\sum_{k=1, k \neq i}^{n} x_{k}+x_{j}\right)}\right) \times \frac{N ! \rho^{-2 n}}{(N-j) !(j-n-1) !} \times e^{-\left(x_{j}+\sum_{k=1, k \neq i}^{n} x_{k}\right) / \rho^{2}} \\
& \times\left(1-e^{-x_{j} / \rho^{2}}\right)^{N-j} \times\left(e^{-x_{j} / \rho^{2}}-e^{-x_{n} / \rho^{2}}\right)^{j-n-1} \\
& \times\left(e^{-x_{i+1} / \rho^{2}}-e^{-x_{i-1} / \rho^{2}}\right) d x_{1} d x_{2} \cdots d x_{i-1} d x_{i+1} \cdots d x_{n} d x_{j}
\end{aligned}
$$


Note that we replace $x_{i}$ with $x_{j}$ in the $Q$ function of the above equation. Similarly, the BER performance can be upperbounded as

$$
\begin{aligned}
\bar{P}_{e b, \mathrm{ECSI}, i j}^{(N, n)}\left(\gamma_{b}\right) \\
\leq \frac{N ! \rho^{-2 n}}{2(N-j) !(j-n-1) !} \\
\quad \times \int_{0}^{\infty} \int_{x_{j}}^{\infty} \int_{x_{n}}^{\infty} \cdots \int_{x_{i+1}}^{\infty} \int_{x_{i-1}}^{\infty} \cdots \int_{x_{2}}^{\infty} e^{-A_{b}\left(x_{j}+\sum_{k=1, k \neq i}^{n} x_{k}\right) / \rho^{2}} \\
\quad \times\left(1-e^{-x_{j} / \rho^{2}}\right)^{N-j}\left(e^{-x_{j} / \rho^{2}}-e^{-x_{n} / \rho^{2}}\right)^{j-n-1} \\
\quad \times\left(e^{-x_{i+1} / \rho^{2}}-e^{-x_{i-1} / \rho^{2}}\right) \\
\quad \times d x_{1} d x_{2} \cdots d x_{i-1} d x_{i+1} \cdots d x_{n} d x_{j} .
\end{aligned}
$$

Integrating continuously $(i-2)$ times in (28) with respect to $x_{1}, x_{2}, \ldots$, and $x_{i-2}$, we arrive at

$$
\begin{aligned}
& \frac{N ! \rho^{-2[n-(i-2)]}}{2(N-j) !(j-n-1) !} \int_{0}^{\infty} \int_{x_{j}}^{\infty} \int_{x_{n}}^{\infty} \cdots \int_{x_{i+1}}^{\infty} e^{-A_{b}\left(x_{j}+\sum_{k=i+1}^{n} x_{k}\right) / \rho^{2}} \\
& \times\left(1-e^{-x_{j} / \rho^{2}}\right)^{N-j} \times\left(e^{-x_{j} / \rho^{2}}-e^{-x_{n} / \rho^{2}}\right)^{j-n-1} \\
& \times\left(e^{-x_{i+1} / \rho^{2}}-e^{-x_{i-1} / \rho^{2}}\right) \\
& \times \frac{e^{-(i-1) A_{b} x_{i-1} / \rho^{2}}}{(i-2) ! A_{b}^{i-2}} d x_{i-1} d x_{i+1} \cdots d x_{n} d x_{j} .
\end{aligned}
$$

Integrating again with respect to $x_{i-1}$ and $x_{i+1}$, we have

$$
\begin{aligned}
& \frac{N ! \rho^{-2(n-i)}}{2(N-j) !(j-n-1) !} \\
& \times \int_{0}^{\infty} \int_{x_{j}}^{\infty} \int_{x_{n}}^{\infty} \cdots \int_{x_{i+3}}^{\infty} e^{-A_{b}\left(x_{j}+\sum_{k=i+2}^{n} x_{k}\right) / \rho^{2}}\left(1-e^{-x_{j} / \rho^{2}}\right)^{N-j} \\
& \times\left(e^{-x_{j} / \rho^{2}}-e^{-x_{n} / \rho^{2}}\right)^{j-n-1} \\
& \times \frac{e^{-\left(i A_{b}+1\right) x_{i+2} / \rho^{2}}}{(i-1) ! A_{b}^{i-1}\left[(i-1) A_{b}+1\right] \cdot\left[i A_{b}+1\right]} d x_{i+2} \cdots d x_{n} d x_{j} .
\end{aligned}
$$

Integrating $(n-i-2)$ times with respect to $x_{i+2}, \ldots, x_{n-1}$, we obtain

$$
\begin{aligned}
& \frac{N ! \rho^{-4}}{2(N-j) !(j-n-1) !} \\
& \times \int_{0}^{\infty} \int_{x_{j}}^{\infty} e^{-A_{b}\left(x_{j}+x_{n}\right) / \rho^{2}}\left(1-e^{-x_{j} / \rho^{2}}\right)^{N-j} \\
& \times\left(e^{-x_{j} / \rho^{2}}-e^{-x_{n} / \rho^{2}}\right)^{j-n-1} \\
& \times \frac{e^{-\left[(n-2) A_{b}+1\right] x_{n} / \rho^{2}}}{(i-1) ! A_{b}^{i-1} \prod_{l=i-1}^{n-2}\left[l A_{b}+1\right]} d x_{n} d x_{j} .
\end{aligned}
$$

We apply binomial expansion over the above expression, namely,

$$
\begin{aligned}
& \left(e^{-x_{j} / \rho^{2}}-e^{-x_{n} / \rho^{2}}\right)^{j-n-1} \\
& \quad=\sum_{r=0}^{j-n-1} C_{j-n-1}^{r} \times e^{-(j-n-r-1) x_{j} / \rho^{2}} \times(-1)^{r} e^{-r x_{n} / \rho^{2}} .
\end{aligned}
$$

Substituting the binomial expansion into (31) and integrating with respect to $x_{n}$, we have

$$
\begin{aligned}
& \frac{N ! \rho^{-2}}{2(N-j) !(j-n-1) !} \\
& \quad \times \int_{0}^{\infty}\left(1-e^{-x_{j} / \rho^{2}}\right)^{N-j} \times \frac{1}{(i-1) ! A_{b} i-1 \prod_{l=i-1}^{n-2}\left[l A_{b}+1\right]} \\
& \quad \times \sum_{r=0}^{j-n-1} C_{j-n-1}^{r} \cdot e^{-(j-n-r-1) x_{j} / \rho^{2}} \times(-1)^{r} \\
& \quad \times \frac{e^{-\left[n A_{b}+r+1\right] x_{j} / \rho^{2}}}{(n-1) A_{b}+r+1} d x_{j} .
\end{aligned}
$$

Applying again the binomial expansion in (33), we arrive at

$$
\begin{aligned}
& \frac{N !}{2(N-j) !(j-n-1) !(i-1) ! A_{b}^{i-1} \prod_{l=i-1}^{n-2}\left(l A_{b}+1\right)} \\
& \times \sum_{m=0}^{N-j} C_{N-j}^{m}(-1)^{m} \times \sum_{r=0}^{j-n-1} C_{j-n-1}^{r}(-1)^{r} \\
& \times \frac{1}{(n-1) A_{b}+r+1} \int_{0}^{\infty} \frac{e^{-\left(n A_{b}+m+j-n\right) x_{j} / \rho^{2}}}{\rho^{2}} d x_{j} .
\end{aligned}
$$


We integrate with respect to $x_{j}$ and attain finally the theoretical expression of BER CUB for the $(N, n)$ MTAS/MISOSTBC system as follows:

$$
\begin{aligned}
& \bar{P}_{e b, \mathrm{ECSI}, i j}^{(N, n)}\left(\gamma_{b}\right) \\
& \leq \frac{N !}{2(N-j) !(j-n-1) !(i-1) ! A_{b}^{i-1} \prod_{l=i-1}^{n-2}\left(l A_{b}+1\right)} \\
& \quad \times \sum_{m=0}^{N-j} C_{N-j}^{m}\left\{\sum_{r=0}^{j-n-1} C_{j-n-1}^{r} \times(-1)^{m+r}\right. \\
& \left.\quad \times \frac{1}{\left[(n-1) A_{b}+r+1\right] \cdot\left[n A_{b}+m+j-n\right]}\right\} .
\end{aligned}
$$

Finally, substituting $A_{b}$ into (35), we arrive at

$$
\begin{aligned}
& \bar{P}_{e b, \mathrm{ECSI}, i j}^{(N, n)}\left(\gamma_{b}\right) \\
& \leq\left(N ! \times\left(2(N-j) !(j-n-1) !(i-1) !\left(C_{n, R_{c}} \rho^{2} \gamma_{b}+1\right)^{i-1}\right.\right. \\
& \left.\left.\times \prod_{l=i-1}^{n-2}\left[l\left(C_{n, R_{c}} \rho^{2} \gamma_{b}+1\right)+1\right]\right)^{-1}\right) \\
& \times \sum_{m=0}^{N-j} C_{N-j}^{m}\left\{\sum_{r=0}^{j-n-1} C_{j-n-1}^{r} \times(-1)^{m+r}\right) \\
& \times\left(1 \times\left(\left[(n-1)\left(C_{n, R_{c}} \rho^{2} \gamma_{b}+1\right)+r+1\right]\right.\right. \\
& \cdot\left[n\left(C_{n, R_{c}} \rho^{2} \gamma_{b}+1\right) A_{b}\right. \\
& \left.\left.+m+j-n])^{-1}\right)\right\} .
\end{aligned}
$$

(II) Exchange between $i=1$ and $x_{j}(j \geqslant n+1)$. In this case, it is easy to examine the theoretical expression of BER CUB as the same as that of exchange between $x_{i}(1<i<n)$ and $x_{j}(j \geqslant n+1)$, namely, (36) including the case of $i=1$.

(III) Exchange between $i=n$ and $x_{j}(j \geqslant n+1)$. In this specific case, similarly, we apply (11); thus the integral formula of system BER is clearly given by

$$
\begin{aligned}
& \bar{P}_{e b, \mathrm{ECSI}, n j}^{(N, n)}\left(\gamma_{b}\right) \\
& =\int_{0}^{\infty} \int_{x_{j}}^{\infty} \int_{x_{n-1}}^{\infty} \cdots \int_{x_{2}}^{\infty} \mathrm{Q}\left(\sqrt{2 C_{n, R_{c}} \gamma_{b}\left(\sum_{k=1}^{n-1} x_{k}+x_{j}\right)}\right) \\
& \quad \times \frac{N ! \rho^{-2 n}}{(N-j) !(j-n) !} \times e^{-\left(x_{j}+\sum_{k=1}^{n-1} x_{k}\right) / \rho^{2}} \times\left(1-e^{-x_{j} / \rho^{2}}\right)^{N-j} \\
& \quad \times\left(e^{-x_{j} / \rho^{2}}-e^{-x_{n-1} / \rho^{2}}\right)^{j-n} d x_{1} d x_{2} \cdots d x_{n-1} d x_{j} .
\end{aligned}
$$

Employing CUB, we have

$$
\begin{aligned}
& \bar{P}_{e b, \mathrm{ECSI}, n j}^{(N, n)}\left(\gamma_{b}\right) \\
& \leq \frac{N ! \rho^{-2 n}}{2(N-j) !(j-n) !} \\
& \quad \times \int_{0}^{\infty} \int_{x_{j}}^{\infty} \int_{x_{n-1}}^{\infty} \cdots \int_{x_{2}}^{\infty} e^{-A_{b}\left(x_{j}+\sum_{k=1}^{n-1} x_{k}\right) / \rho^{2}} \times\left(1-e^{-x_{j} / \rho^{2}}\right)^{N-j} \\
& \quad \times\left(e^{-x_{j} / \rho^{2}}-e^{-x_{n-1} / \rho^{2}}\right)^{j-n} d x_{1} d x_{2} \cdots d x_{n-1} d x_{j} .
\end{aligned}
$$

Integrating $(n-2)$ times in the above integral formula with respect to $x_{1}, \ldots, x_{n-2}$, we obtain

$$
\begin{aligned}
& \frac{N ! \rho^{-4}}{2(N-j) !(j-n) !} \\
& \times \int_{0}^{\infty} \int_{x_{j}}^{\infty} e^{-A_{b}\left(x_{j}+x_{n-1}\right) / \rho^{2}} \times\left(1-e^{-x_{j} / \rho^{2}}\right)^{N-j} \\
& \times\left(e^{-x_{j} / \rho^{2}}-e^{-x_{n-1} / \rho^{2}}\right)^{j-n} \\
& \times \frac{1}{(n-2) ! A_{b}^{n-2}} e^{-(n-2) A_{b} x_{n-1} / \rho^{2}} d x_{n-1} d x_{j} .
\end{aligned}
$$

Similarly, expanding the two binomials in the above integral formula, then integrating with respect to $x_{n-1}$, and cleaning it up, we arrive at

$$
\begin{aligned}
& \frac{N ! \rho^{-2}}{2(N-j) !(j-n) !} \times \sum_{m=0}^{N-j} C_{N-j}^{m} \times(-1)^{m} \\
& \times \int_{0}^{\infty} e^{-\left(A_{b}+m\right) x_{j} / \rho^{2}} \times \sum_{r=0}^{j-n} C_{j-n}^{r} \times(-1)^{r} \\
& \times e^{-(j-n-r) x_{j} / \rho^{2}} \times \frac{1}{(n-2) ! A_{b}^{n-2}} \times \frac{e^{\left[(n-1) A_{b}+r\right] x_{j} / \rho^{2}}}{(n-1) A_{b}+r} d x_{j} .
\end{aligned}
$$

Finally, we integrate with respect to $x_{j}$ and derive the theoretical expression of system BER CUB for the case of exchange between $x_{n}$ and $x_{j}(j \geqslant n+1)$ as follows:

$$
\begin{aligned}
\bar{P}_{e b, \mathrm{ECSI}, n j}^{(N, n)}\left(\gamma_{b}\right) \\
\leq \frac{N !}{2(N-j) !(j-n) !(n-2) ! A_{b}^{n-2}} \\
\quad \times \sum_{m=0}^{N-j} \sum_{r=0}^{j-n}(-1)^{m+r} \frac{C_{N-j}^{m} C_{j-n}^{r}}{\left[(n-1) A_{b}+r\right] \cdot\left[n A_{b}+j+m-n\right]} .
\end{aligned}
$$


Substituting $A_{b}$ into (41), we obtain

$$
\begin{aligned}
& \bar{P}_{e b, \mathrm{ECSI}, n j}^{(N, n)}\left(\gamma_{b}\right) \\
& \leq \frac{N !}{2(N-j) !(j-n) !(n-2) !\left(C_{n, R_{c}} \rho^{2} \gamma_{b}+1\right)^{n-2}} \\
& \quad \times \sum_{m=0}^{N-j} \sum_{r=0}^{j-n}(-1)^{m+r} \\
& \quad \times\left(C_{N-j}^{m} C_{j-n}^{r} \times\left(\left[(n-1)\left(C_{n, R_{c}} \rho^{2} \gamma_{b}+1\right)+r\right]\right.\right. \\
& \left.\left.\cdot\left[n\left(C_{n, R_{c}} \rho^{2} \gamma_{b}+1\right)+j+m-n\right]\right)^{-1}\right) .
\end{aligned}
$$

3.4. Average BER. Let the correct probability of CSI be $P_{c}$; then the error probability that reveals exchange of two ordinal numbers is $\left(1-P_{c}\right)$. We further assume that the exchanges are uniform distribution; namely, they have equal probability with $P_{\mathrm{err}}=2\left(1-P_{c}\right) / N(N-1)$. In the case of the ICSI, the BER of the $(N, n)$ system is denoted by $\mathrm{BER}_{\mathrm{ICSI}}$, and the case of ECSI, the BER of the resulting subset $\mathbf{X}_{n}^{\prime}$ by $\mathrm{BER}_{\text {ECSI}}$. Since $n(n-1) / 2+(N-n)(N-n-1) / 2$ selection subsets do not affect the system BER performance and other $n(N-n)$ selection subsets are to affect the BER performance, the resulting BERs are denoted by $\quad \mathrm{BER}_{\mathrm{ECSI}, 1(n+1)}, \ldots, \mathrm{BER}_{\mathrm{ECSI}, 1 N}, \ldots, \mathrm{BER}_{\mathrm{ECSI}, n(n+1)}, \ldots$, $\mathrm{BER}_{\mathrm{ECSI}, n N}$, respectively. Thus, the average BER of the $(N, n)$ MTAS/MISO-STBC system is given by

$$
\begin{aligned}
\overline{\mathrm{BER}}= & P_{c} \times \mathrm{BER}_{\mathrm{ICSI}}+P_{\mathrm{err}} \times \mathrm{BER}_{\mathrm{ICSI}} \\
& \times \frac{[n(n-1)+(N-n)(N-n-1)]}{2} \\
& +P_{\mathrm{err}} \times \sum_{i=1}^{n} \sum_{j=n+1}^{N} \mathrm{BER}_{\mathrm{ECSI}, i j .}
\end{aligned}
$$

The right side of the above expression terms BER $_{\text {ICSI }}$ and $\mathrm{BER}_{\mathrm{ECSI}, i j}$ is replaced with the denotations in (26), (36), and (42). Thus we can arrive at

$$
\begin{aligned}
& \overline{\mathrm{BER}} \\
& =\bar{P}_{e b, \mathrm{ICSI}}^{(N, n)}\left(\gamma_{b}\right)\left\{P_{c}+P_{\mathrm{err}}\right. \\
& \left.\quad \times \frac{[n(n-1)+(N-n)(N-n-1)]}{2}\right\} \\
& +P_{\mathrm{err}} \times \sum_{i=1}^{n} \sum_{j=n+1}^{N} \bar{P}_{e b, \mathrm{ECSI}, i j}^{(N, n)}\left(\gamma_{b}\right) .
\end{aligned}
$$

Substituting right side of the Inequalities (26), (36) and (42) into the right side of (44), we can obtain the theoretical expression of the BER CUB for the $(N, n)$ MTAS/MISOSTBC system.

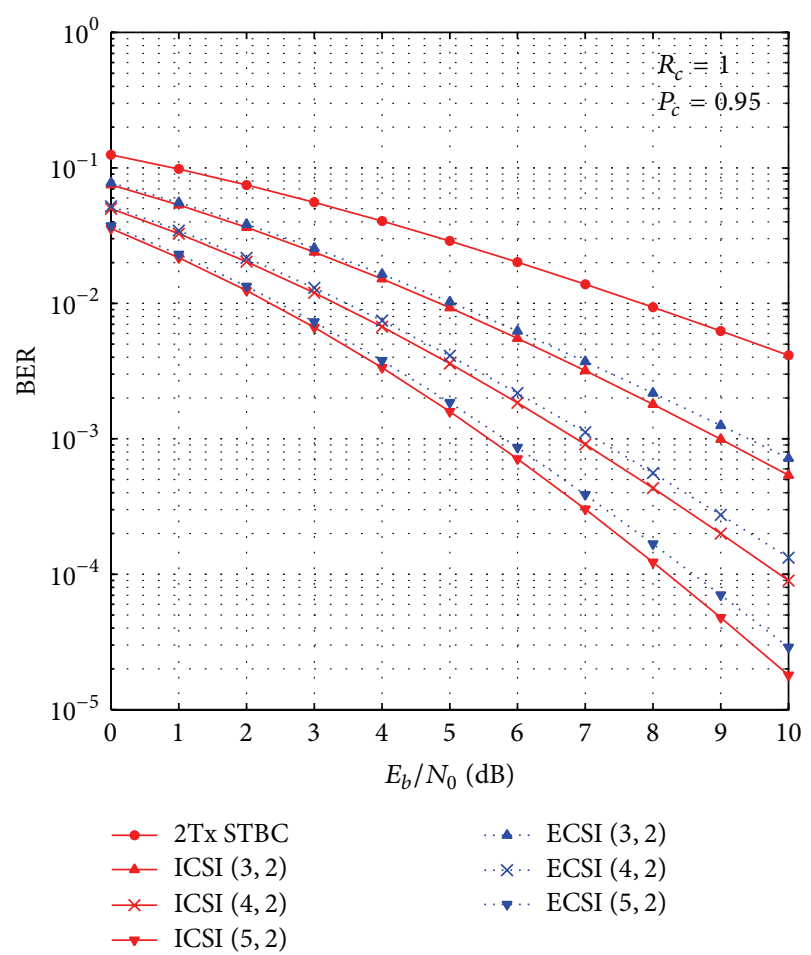

Figure 2: $P_{c}=0.95$, code rate $R_{c}=1$, and $n=2, N=2,3,4$, and 5, the curves of BER CUB as a function of $E_{b} / N_{0}$.

\section{Numerical and Simulating Results}

In our computer simulation and analysis, we use the system model depicted in Figure 1. We assume that the wireless channel is independent, uncorrelated, and flat fading Rayleigh distribution with Gaussian variance $\sigma^{2}=0.5$. Applying (18), (26), (36), and (42), let the correct probability of CSI at the transmit side $P_{c}=0.95$. If the number of the selected antennas $n$ is fixed, we change the number of the available transmit antennas $N$. In Figures 2, 3, and 4, we present the curves of BER CUB as a function of $E_{b} / N_{0}$ for the three cases of STBC code rate $R_{c}=1,3 / 4$, and $1 / 2$ over STBCMISO/MTAS system, respectively. In Figure 2, if the number of the selected antennas $n$ is fixed 2, then the system always chooses the best two antennas to transmit encoding Alamouti scheme data streams with full code rate. The number of the available transmit antennas is $N=2,3,4$ and 5 , respectively. Let the number of the selected antennas be $n=3$, and the number of the available transmit antennas be $N=3,4,5$, and 6. We utilize STBC with code rate $R_{c}=3 / 4$ in Figure 3, and $R_{c}=1 / 2$ in Figure 4 for the three transmit antennas, respectively. We can see from the three curve figures of BER CUB, whether the CSI is perfect or imperfect, in the condition of the fixed transmit antennas. Compared with no antenna selection, the system employing multiple antennas selection technique can enhance markedly BER performance. In addition, in the presence of the ECSI at the transmitter side, we also observe in the three figures that the BER curves are up a little. It is coincident with our expectation. However, this 


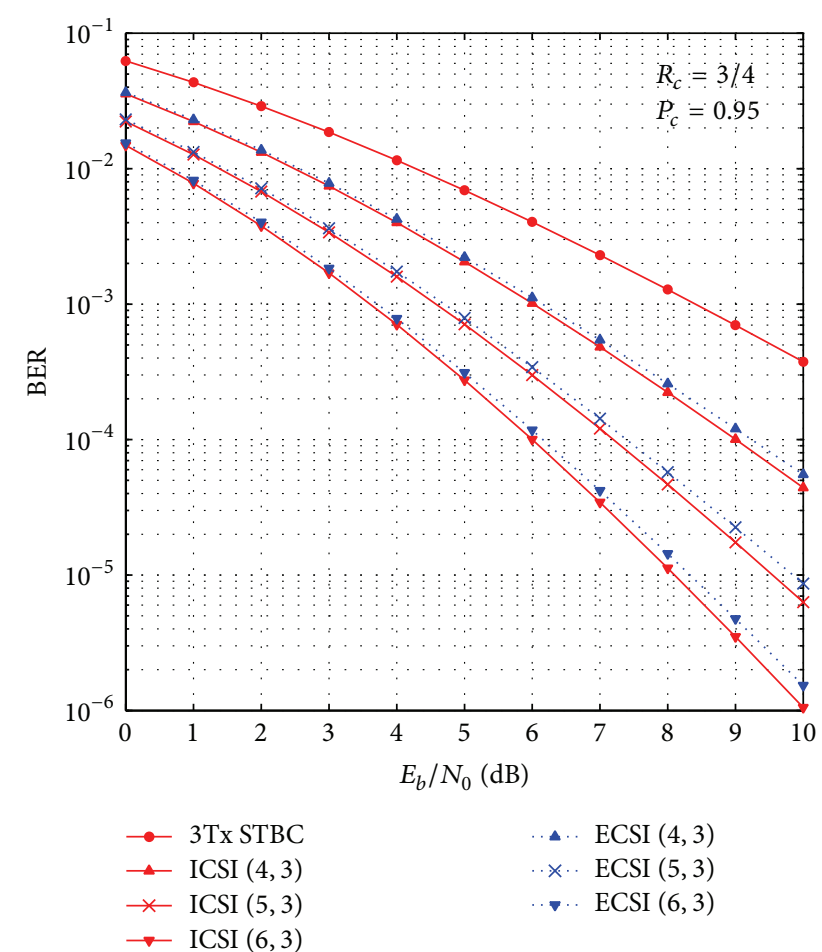

Figure 3: $P_{c}=0.95$, code rate $R_{c}=3 / 4$, and $n=3, N=3,4,5$, and 6 , the curves of BER CUB as a function of $E_{b} / N_{0}$.

effect of ECSI on the BER performance for the $(N, n)$ MTAS system is smaller when $P_{c}$ approaches 1; that is, $P_{c}=0.95$.

In order to examine the relationship of the BER performance between MTAS and full complexity system, let the number of the available selected transmit antennas $N$ be fixed 5 , and the number of the selected antennas $n$ be equal to 2 , 3 , and 4 , respectively. Figure 5 plots the curves of the BER CUB as a function of $E_{b} / N_{0}$ at the range of $0 \sim 10 \mathrm{~dB}$. For the two cases $n=3$ and 4 ; we consider STBCs with both code rates $1 / 2$ and $3 / 4$. For comparison, we also present the BER performance curves for the full complexity system with number of the transmit antennas $N=5$ in Figure 5. When $n$ gets a larger value, we observe that the BER magnitude increase is not very remarkable. Therefore, we can observe from Figure 5 that a reasonable value of $n$, that is, 2 and/or 3 , should be chosen in practical applications. In addition, we can also see that the system BER performance has approached that of the full complexity in this case. In a whole encoding period of STBC, at the condition of same BER, let transmit antennas number be $n=3$ and 4 . The BER with $3 / 4$ code rate is $3 \mathrm{~dB}$ low compared with half code rate; namely, there are $3 \mathrm{~dB}$ gap between the BER curves of half rate and that of $3 / 4$ rate for the two cases of $(5,3)$ and $(5,4)$.

In order to observe the effects of the CSI correct probability $P_{c}$ on BER performance, in Figure 6 , let $E_{b} / N_{0}=5 \mathrm{~dB}$ and $n=2$. We plot the curves of BER as a function of $P_{c}$ for three cases of $(3,2),(4,2)$, and $(5,2)$, respectively. The results show in the three figures that the MTAS system can still maintain better BER performance when $P_{c} \geq 0.9$; however, when $P_{c}<$ 0.9, the BER performance reduces fast. Therefore, when $P_{c}$

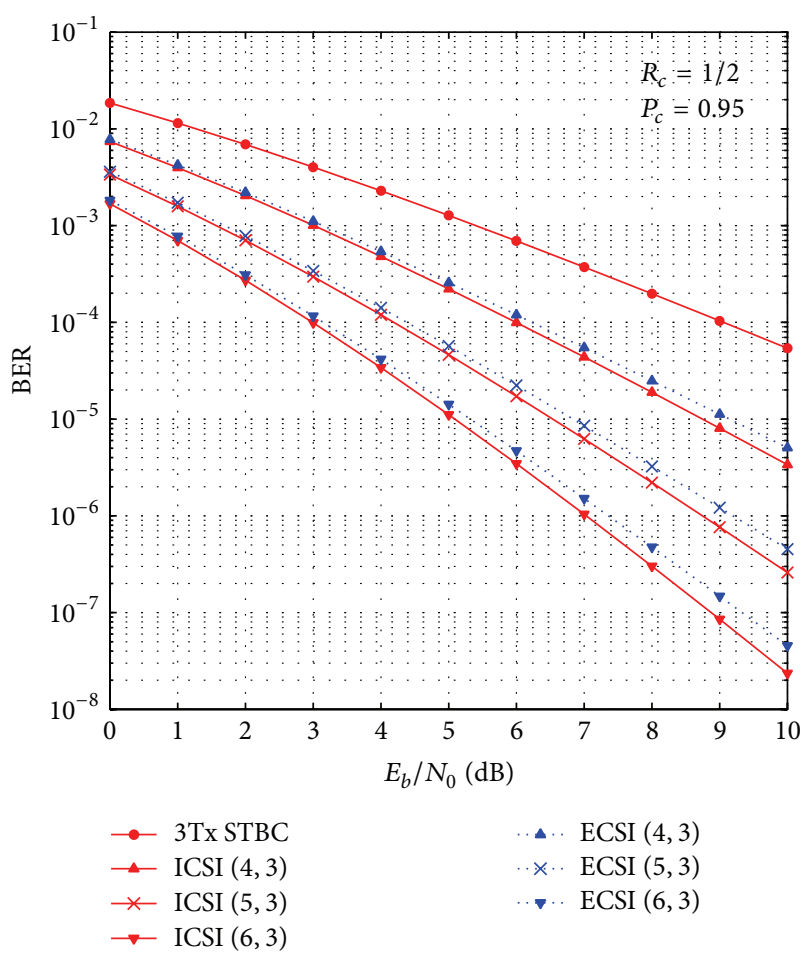

Figure 4: $P_{c}=0.95$, code rate $R_{c}=1 / 2$, and $n=3, N=3,4,5$, and 6 , the curves of BER CUB as a function of $E_{b} / N_{0}$.

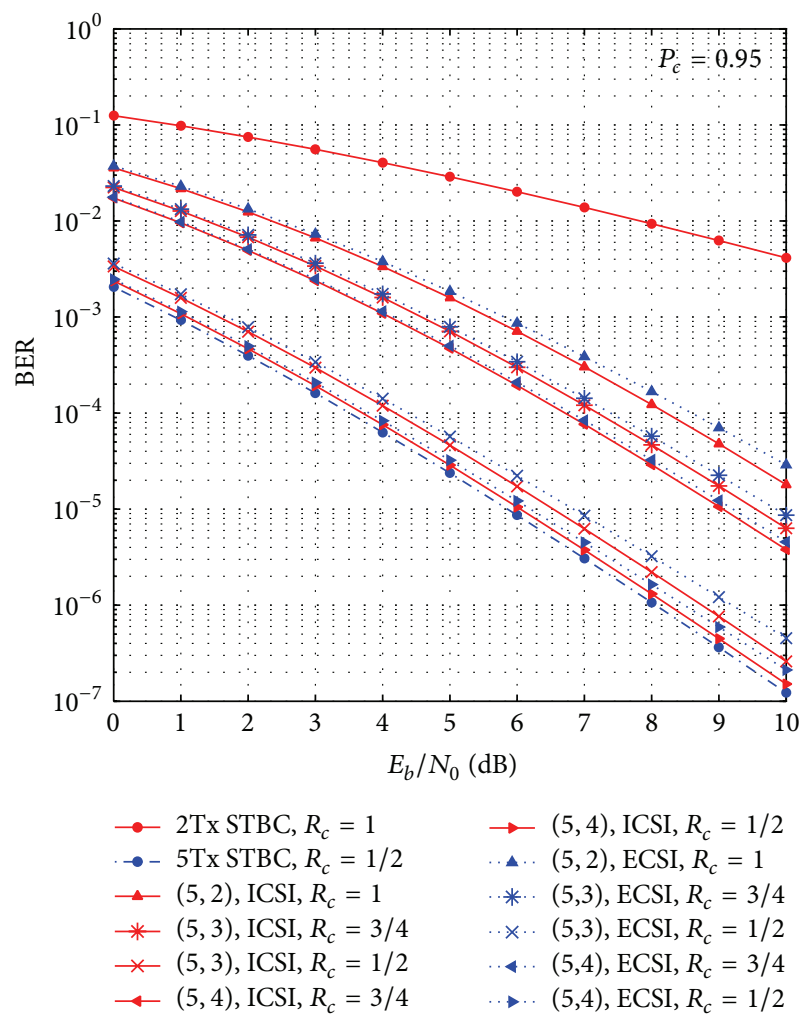

Figure 5: $P_{c}=0.95$, code rate $R_{c}=1,1 / 2$, and $3 / 4, N=5, n=2,3$, and 4 , the curves of BER CUB as a function of $E_{b} / N_{0}$. 


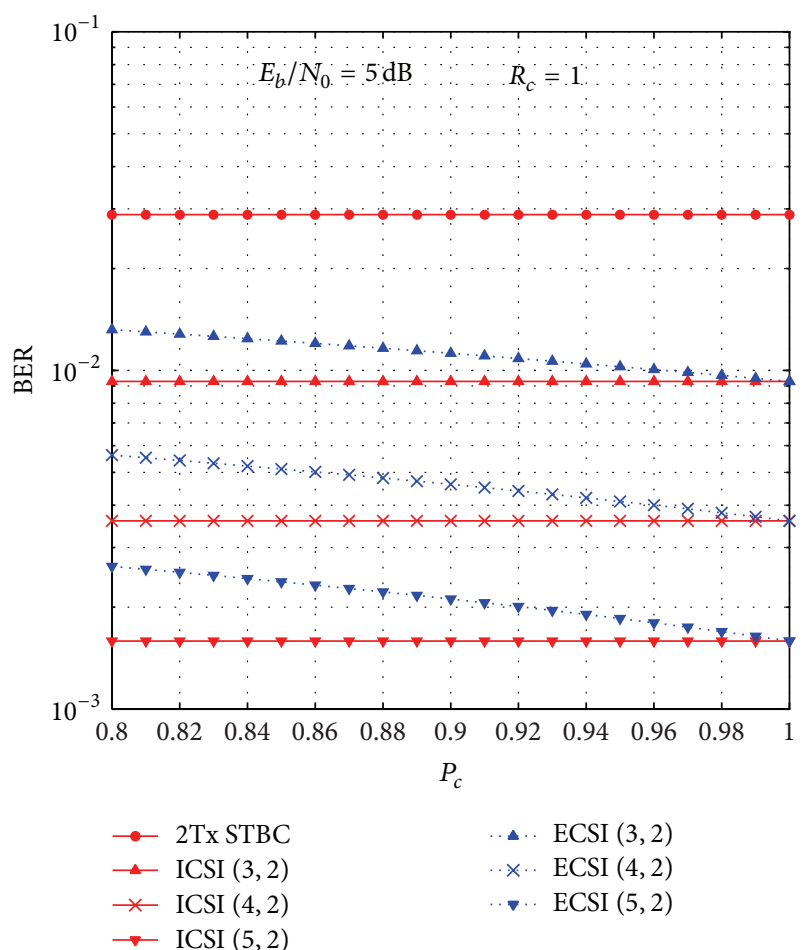

FIgURE 6: $E_{b} / N_{0}=5 \mathrm{~dB}$ and $n=2$, the curves of BER as a function of $P_{c}$ in the three cases of $(3,2),(4,2)$, and $(5,2)$.

takes a less value, the effects of ECSI on the BER performance should get our attention. In addition, when $n=2$, we also observe such a phenomenon that, in the condition of the same $P_{c}$, the differential values between the ECSI BER and the ICSI BER increase as the available number $N$ of the selected transmit antennas increases. The cause can be explained as along with the available number of antenna increasing, the probability of severe fading channel increase. It is possible to have exchange between the best antenna and the worst antenna, thus resulting in the rise of BER value.

At last, in order to validate and support our theoretical analyses, in the case of $n=2$, we perform extensive simulations with Monte-Carlo method (MCM). At first,we consider the case of inerrable CSI. Figure 7 depicts the BER results with Monte-Carlo simulation (MCS) for the four cases of $(2,2),(3,2),(4,2)$, and $(5,2)$, respectively. For comparison, in the figure, we also plot the curves of the BER CUB for the expressions (18), (26), (36), and (42). We observe from Figure 7 that all BER performance curves have the same degree of slope. Moreover, the gaps between the simulated curves and the analytical curves are little. Furthermore, Figure 7 also shows that our analytical results are a tighter upper bound. Therefore, the theoretical expressions proposed in this correspondence make an important implication in practical applications. Let $P_{c}=0.9$. Figure 8 depicts the BER results with MCS for the three cases of $(3,2),(4,2)$, and $(5,2)$, respectively. Similarly, for comparison, we also give the true values of BER with MCS for the case of ICSI. No less than the Figures 2, 3, 4, and 5, along with number of available transmit antennas $N$ increasing, the BER performance will become

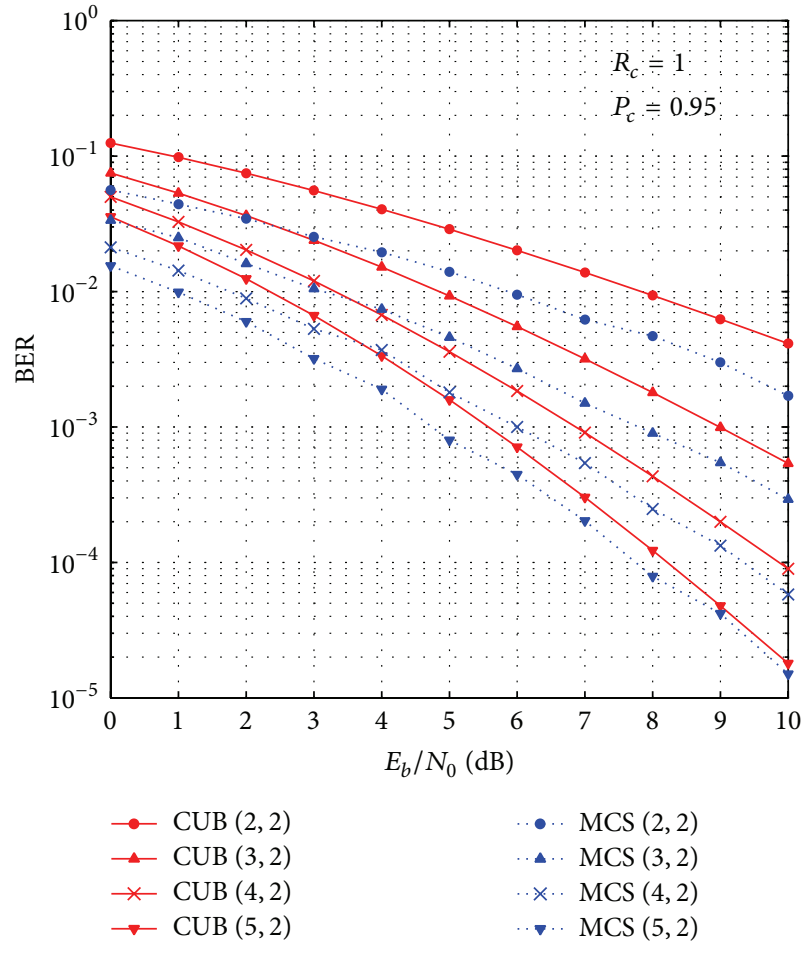

FIGURE 7: Performance comparison between simulating values and CUB for the four cases of $(2,2),(3,2),(4,2)$, and $(5,2)$, respectively.

better. Similarly, relative to the ICSI cases, the simulated BER results of ECSI increase a little more than ICSI does.

Finally, let $P_{c}=0.9$ and the selected number of transmit antennas $n=2$. Similarly, comparing the simulated BER values with that of analytical CUB in the same figure, for the two cases of ICSI and ECSI, Figure 9 plots the BER performance curves of both MCM and CUB for the four cases of $(2,2),(3,2),(4,2)$, and $(5,2)$, respectively. The results also indicate that our BER CUB performance analyses over STBC-MISO/MTAS system are consistent with the practical situations. The MTAS technique can enhance largely the performance of the wireless link.

\section{Conclusions}

MIMO system is a promising technique in future wireless mobile communication. However, among the implications of employing multiple antennas is the considerable increase in the cost of the additional hardware required in the form of RF chains, as well as the physical limitation of wireless devices. Therefore, recently, the investigations of MTAS/MIMO systems have become an explosion of interest. At present, the concerning papers are quite scarce. Therefore, this work focuses mainly on the multiple-transmit antenna selection of MIMO system with STBC. In order to see the effects of ECSI on the BER performance for $(N, n)$ MTAS/MISO-STBC system, the correspondence suggests a theoretical model for multiple-transmit antennas selection system considering ECSI at the transmitter side. The analytical expressions of the BER CUB derive in theory for three STBC code 


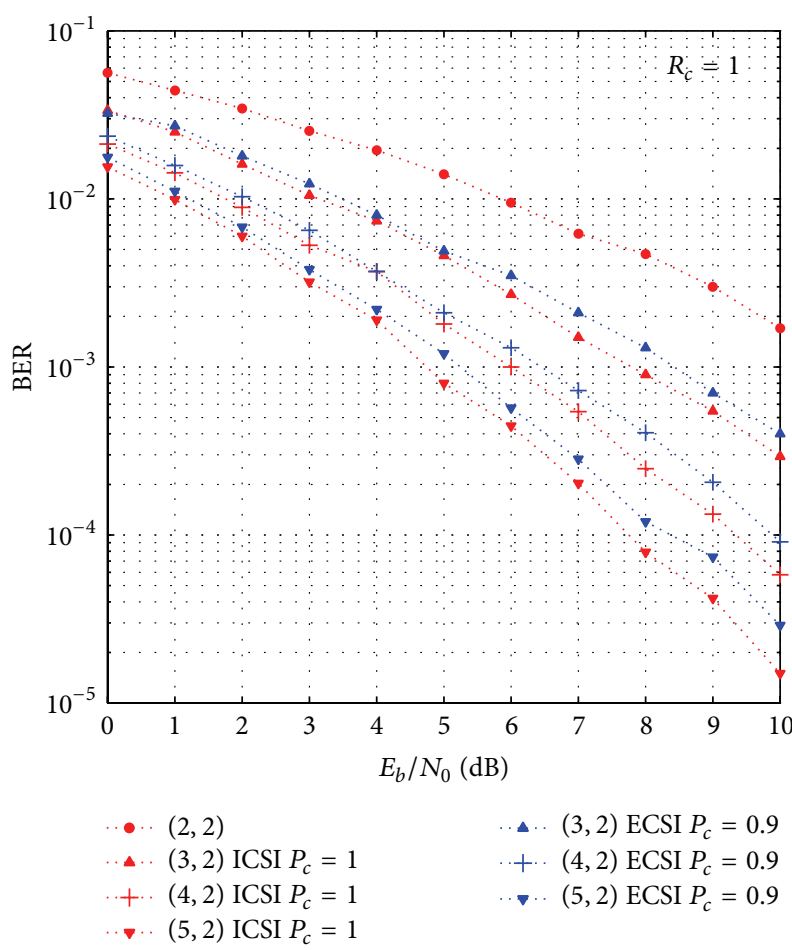

FIgURE 8: In the case of $P_{c}=0.9$, BER performance comparison between ICSI and ECSI with MCS for the three cases of $(3,2),(4,2)$, and $(5,2)$, respectively.

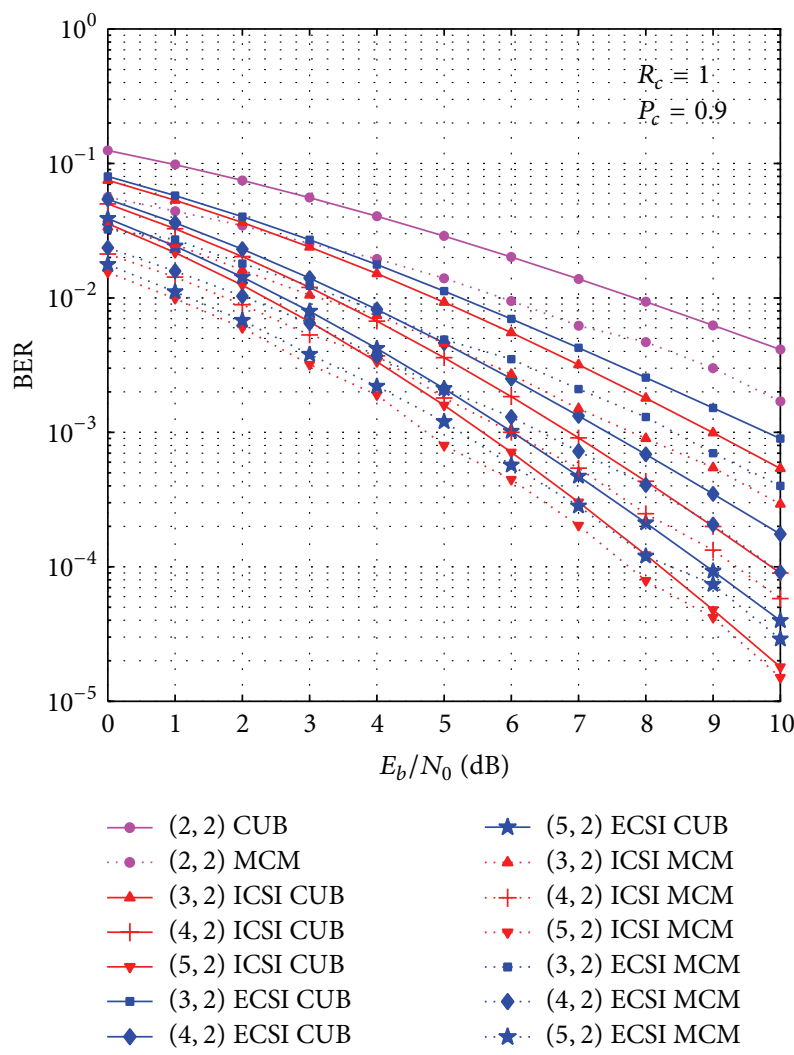

FIgURE 9: Let $P_{c}=0.9$ and $n=2$, in the two case of ICSI and ECSI, BER performance curves of both MCM and CUB for the four cases of $(2,2),(3,2),(4,2)$, and $(5,2)$, respectively. rates. Through extensive Monte-Carlo simulation, the results validate and support our analysis. In addition, we also come to some beneficial conclusions. Compared with the full complexity system (no MTAS), the investigation shows that the MISO-STBC system employing MTAS is sure of improving largely the BER performance. Moreover, it also mitigates effectively the effects of ECSI. These results will provide a theoretical guidance for practical MIMO system applications.

\section{Conflict of Interests}

The author declares that there is no conflict of interests regarding the publication of this paper.

\section{Acknowledgments}

This paper is supported by the Natural Science Foundation of Fujian Province of China (no. 2012J01270), by the Technology Research and Development Projects of Quanzhou city (2012Z82), and by the Natural Science Foundation of Huaqiao University (Excellent Level Talents) (no. 11BS430).

\section{References}

[1] N. Seshadri and J. H. Winters, "Two signaling schemes for improving the error performance of frequency division duplex (FDD) transmission systems using transmitter antenna diversity," International Journal of Wireless Information Networks, vol. 1, no. 1, pp. 49-60, 1994.

[2] V. Tarokh, N. Seshadri, and A. R. Calderbank, "Space-time codes for high data rate wireless communication: performance criterion and code construction," IEEE Transactions on Information Theory, vol. 44, no. 3, pp. 744-765, 1998.

[3] V. Tarokh, H. Jafarkhani, and A. R. Calderbank, "Space-time block codes from orthogonal designs," IEEE Transactions on Information Theory, vol. 45, no. 5, pp. 1456-1467, 1999.

[4] A. Paulraj and T. Kailath, "Increasing capacity in wireless broadcast systems using distributed transmission/directional reception," U. S. Patent 345 599, 1994.

[5] E. Telatar, "Capacity of multi-antenna Gaussian channels," European Transactions on Telecommunications, vol. 10, no. 6, pp. 585-595, 1999.

[6] G. J. Foschini, "Layered space-time architecture for wireless communication in a fading environment when using multielement antennas," Bell Labs Technical Journal, vol. 1, no. 2, pp. 41-59, 1996.

[7] G. J. Foschini and M. J. Gans, "On limits of wireless communications in a fading environment when using multiple antennas," Wireless Personal Communications, vol. 6, no. 3, pp. 311-335, 1998.

[8] J. H. Winters, "On the capacity of radio communications systems with diversity in Rayleigh fading environments," IEEE Journal on Selected Areas in Communications, vol. 5, no. 5, pp. 871-878, 1987.

[9] M. Max, Multi-Antenna Digital Radio Transmission, Artech House, Boston, Mass, USA, 2001.

[10] D. A. Gore, R. U. Nabar, and A. Paulraj, "Selecting an optimal set of transmit antennas for a low rank matrix channel," in Proceedings of the IEEE Interntional Conference on Acoustics, 
Speech, and Signal Processing (ICASSP '00), pp. 2785-2788, Istanbul, Turkey, June 2000.

[11] S. Sandhu, R. U. Nabar, D. A. Gore, and A. Paulraj, "Nearoptimal selection of transmit antennas for a MIMO channel based on Shannon capacity," in Proceedings of the 34th Asilomar Conference on Signals, Systems and Computer, vol. 1, pp. 567-571, Pacific Grove, Calif, USA, November 2000.

[12] A. F. Molisch, M. Z. Win, and J. H. Winters, "Capacity of MIMO systems with antenna selection," in Proceedings of the IEEE International Conference on Communications (ICC '01), pp. 570574, Helsinki, Finland, June 2001.

[13] R. W. Heath and A. Paulraj, "Antenna selection for spatial multiplexing systems based on minimum error rate," in Proceedings of the International Conference on Communications (ICC '01), pp. 2276-2280, Helsinki, Finland, June 2000.

[14] D. Gore and A. Paulraj, "Space-time block coding with optimal antenna selection," in Proceedings of the IEEE Interntional Conference on Acoustics, Speech, and Signal Processing (ICASSP '01), pp. 2441-2444, Salt Lake City, UT, USA, May 2001.

[15] A. Gorokhov, "Antenna selection algorithms for MEA transmission systems," in Proceedings of the IEEE International Conference on Acoustic, Speech, and Signal Processing (ICASSP '02), pp. III-2857-III-2860, Orlando, Fla, USA, May 2002.

[16] M. Gharavi-Alkhansari and A. B. Gershman, "Fast antenna subset selection in MIMO systems," IEEE Transactions on Signal Processing, vol. 52, no. 2, pp. 339-347, 2004.

[17] D. Gore and A. Paulraj, "Statistical MIMO antenna subset selection with space-time coding," in Proceedings of the International Conference on Communications (ICC '02), pp. 641645, New York, NY, USA, May 2002.

[18] A. Gorokhov, D. A. Gore, and A. J. Paulraj, "Receive antenna selection for MIMO spatial multiplexing: theory and algorithms," IEEE Transactions on Signal Processing, vol. 51, no. 11, pp. 2796-2807, 2003.

[19] R. S. Blum and J. H. Winters, "On optimum MIMO with antenna selection," IEEE Communications Letters, vol. 6, no. 8, pp. 322-324, 2002.

[20] I. Berenguer and X. Wang, "MIMO antenna selection with lattice-reduction-aided linear receivers," IEEE Transactions on Vehicular Technology, vol. 53, no. 5, pp. 1289-1302, 2004.

[21] A. F. Molisch, "MIMO systems with antenna selection-an overview," in Proceedings of the Radio and Wireless Conference (RAWCON '03), pp. 167-170, Boston, Mass, USA, August 2003.

[22] D. A. Gore and A. J. Paulraj, "MIMO antenna subset selection with space-time coding," IEEE Transactions on Signal Processing, vol. 50, no. 10, pp. 2580-2588, 2002.

[23] M. Z. Win and J. H. Winters, "Virtual branch analysis of symbol error probability for hybrid selection/maximal-ratio combining in rayleigh fading," IEEE Transactions on Communications, vol. 49, no. 11, pp. 1926-1934, 2001.

[24] A. Ghrayeb and T. M. Duman, "Performance analysis of MIMO systems with antenna selection over quasi-static fading channels," IEEE Transactions on Vehicular Technology, vol. 52, no. 2, pp. 281-288, 2003.

[25] I. Bahceci, T. M. Duman, and Y. Altunbasak, "Antenna selection for multiple-antenna transmission systems: performance analysis and code construction," IEEE Transactions on Information Theory, vol. 49, no. 10, pp. 2669-2681, 2003.

[26] W. H. Wong and E. G. Larsson, "Orthogonal space-time block coding with antenna selection and power allocation," Electronics Letters, vol. 39, no. 4, pp. 379-381, 2003.
[27] X. N. Zeng and A. Ghrayeb, "Performance bounds for spacetime block codes with receive antenna selection," IEEE Transactions on Information Theory, vol. 50, no. 9, pp. 2130-2137, 2004.

[28] Z. Chen, J. Yuan, B. Vucetic, and Z. Zhou, "Performance of Alamouti scheme with transmit antenna selection," Electronics Letters, vol. 39, no. 23, pp. 1666-1668, 2003.

[29] Z. Chen, B. Vucetic, and J. Yuan, "Space-time trellis codes with transmit antenna selection," Electronics Letters, vol. 39, no. 11, pp. 854-855, 2003.

[30] H. Jafarkhani, "A quasi-orthogonal space-time block code," IEEE Transactions on Communications, vol. 49, no. 1, pp. 1-4, 2001.

[31] E. G. Larsson and P. Stoica, Space-Time Block Coding for Wireless Communications, Cambridge University Press, Cambridge, UK, 2003.

[32] O. Tirkkonen, "Optimizing space-time block codes by constellation rotations," in Proceedings of the Finnish Wireless Communication Workshop (FWCW '01), p. 5940, Tampere, Finland, October 2001.

[33] A. Sezgin and E. A. Jorswieck, "On optimal constellations for quasi-orthogonal space-time codes," in Proceedings of the IEEE International Conference on Accoustics, Speech, and Signal Processing, pp. 345-348, April 2003.

[34] A. Sezgin, E. A. Jorswieck, and H. Boche, "Performance criteria analysis and further performance results for quasi-orthogonal space-time block codes," in Proceedings of the 3rd IEEE International Symposium on Signal Processing and Information Technology (ISSPIT '03), pp. 102-105, Darmstadt, Germany, July 2003.

[35] B. Hassibi and B. M. Hochwald, "High-rate codes that are linear in space and time," IEEE Transactions on Information Theory, vol. 48, no. 7, pp. 1804-1824, 2002.

[36] A. Sezgin, E. A. Jorswieck, and E. Costa, "LDC in MIMO Ricean channels: optimal transmit strategy with MMSE detection," IEEE Transactions on Signal Processing, vol. 56, no. 1, pp. 313328, 2008.

[37] B. C. Arnold, N. Balakrishnan, and H. N. Nagaraja, A First Course in Order Statistics, Wiley-Interscience, New York, NY, USA, 1992.

[38] J. G. Proakis, Digital Communications, McGraw-Hill, New York, NY, USA, 4th edition, 2000. 

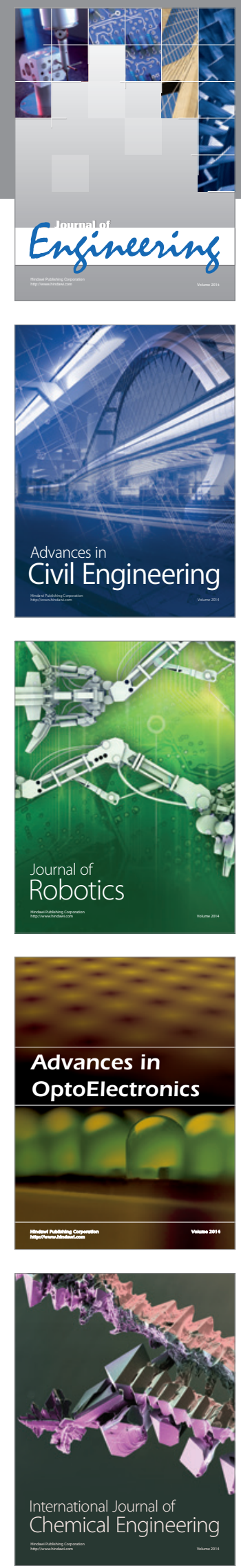

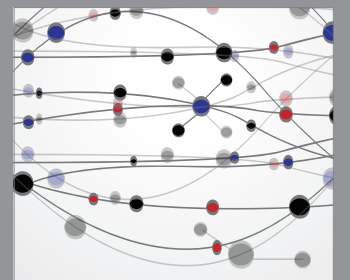

The Scientific World Journal
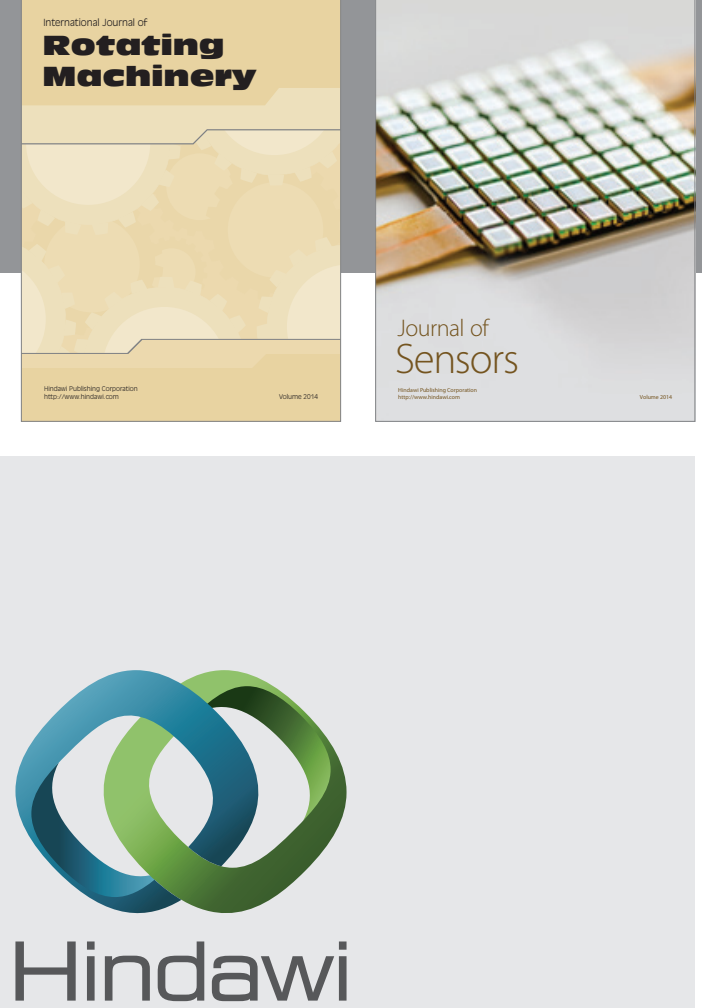

Submit your manuscripts at http://www.hindawi.com
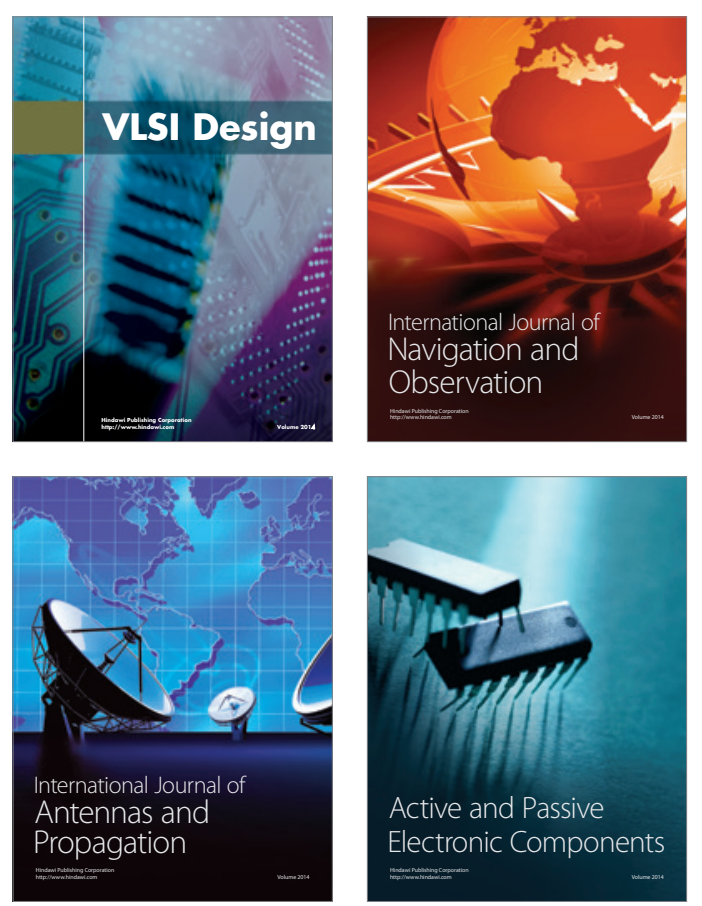
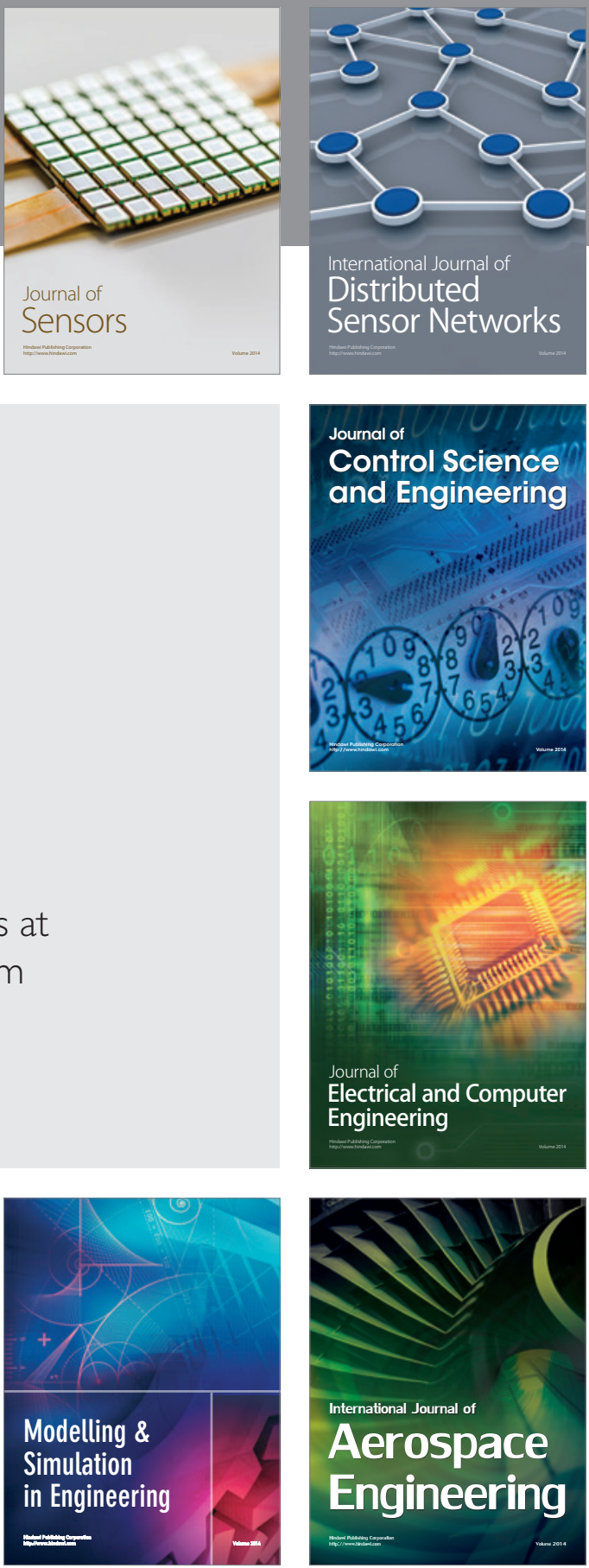

Journal of

Control Science

and Engineering
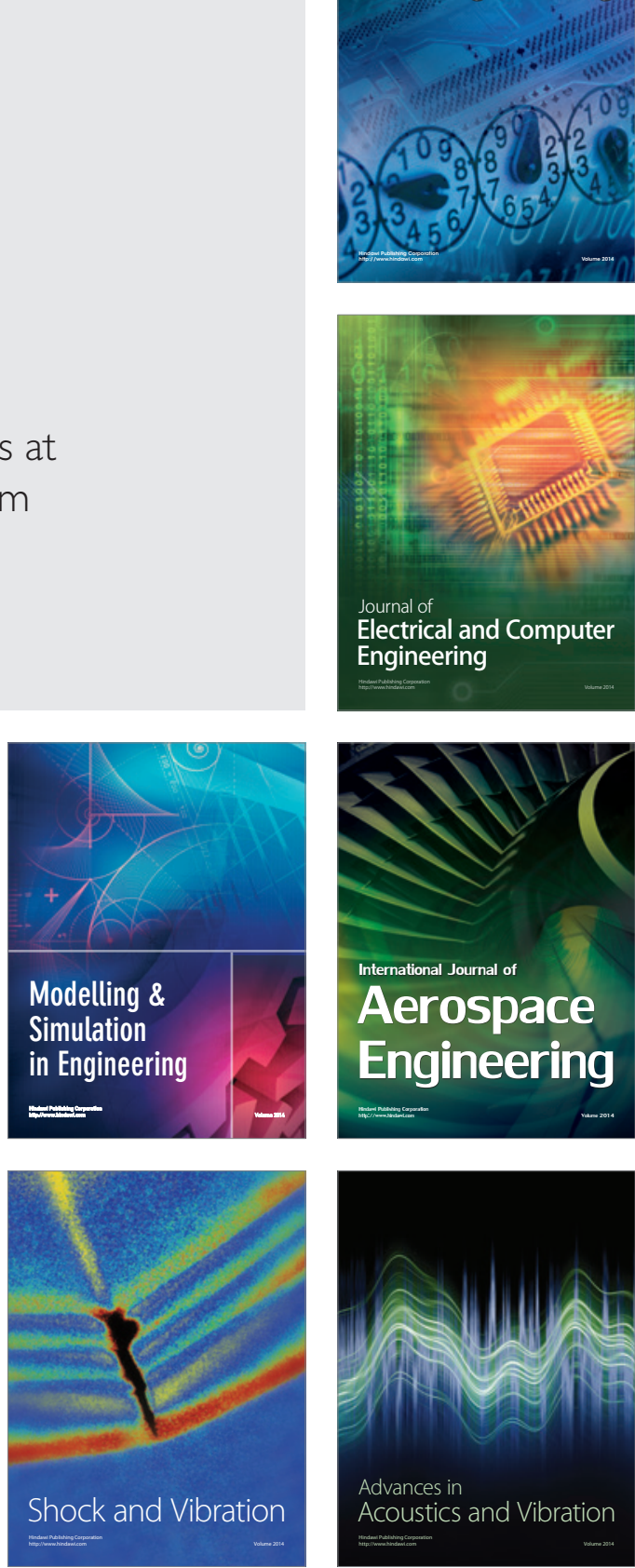